\title{
artigo
}

\section{Atenção à população em situação de rua no âmbito do sistema único de saúde: uma revisão sistemática com metassíntese}

Attention to the population in street situation under the unique health system: a systematic review with meta-synthesis Atención a la población en la situación de la calle bajo el sistema de salud único: una revisión sistemática con metasíntesis

\section{RESUMO}

Objetivo: Analisar o acesso aos serviços de saúde e se há discriminações no atendimento à população em situação de rua. Método: Trata-se de uma revisão sistemática da literatura com metassíntese, utilizando o protocolo PRISMA para a busca e utilização de estudos, facilitando a avaliação detalhada de revisões publicadas. O levantamento dos dados ocorreu entre os meses de outubro de 2019 e abril de 2020, através das bases de dados SciELO, MEDLINE, LILACS e BDENF. Resultados: A população em situação de rua sofre rejeição no atendimento aos serviços públicos de saúde e o acesso de entrada e constância nesses serviços, é um direito legal, contudo, está sendo negado. Conclusão: É possível evidenciar obstáculos no acesso aos serviços do Sistema Único de Saúde, sobretudo por falta de documentos de identificação, além de existirem discriminações durante o atendimento nesses serviços. Há ainda fragilidades nas políticas públicas referentes às necessidades deste público, gerando injustiças ao desrespeitar seus direitos e necessidades.
\end{abstract}

DESCRITORES: População em Situação de Rua; Saúde; Saúde Pública.

\section{ABSTRACT}

Objective: To analyze access to health services and whether there is discrimination in serving the homeless population. Method: This is a systematic review of the literature with meta-synthesis, using the PRISMA protocol for the search and use of studies, facilitating the detailed evaluation of published reviews. The data survey took place between the months of October 2019 and April 2020, using the databases SciELO, MEDLINE, LILACS and BDENF. Results: The population living on the street suffers rejection when attending public health services and access to and access to these services is a legal right, however, it is being denied. Conclusion: It is possible to evidence obstacles in accessing the services of the Unified Health System, mainly due to the lack of identification documents, in addition to the existence of discrimination while attending these services. There are also weaknesses in public policies regarding the needs of this public, creating injustices when they disrespect their rights and needs. DESCRIPTORS: Homeless Persons; Health; Public Health.

\section{RESUMEN}

Objetivo: analizar el acceso a los servicios de salud y si existe discriminación en el servicio a la población sin hogar. Método: Esta es una revisión sistemática de la literatura con meta-síntesis, utilizando el protocolo PRISMA para la búsqueda y uso de estudios, facilitando la evaluación detallada de las revisiones publicadas. La recopilación de datos tuvo lugar entre los meses de octubre de 2019 y abril de 2020, utilizando las bases de datos SciELO, MEDLINE, LILACS y BDENF. Resultados: La población que vive en la calle sufre rechazo cuando asiste a los servicios de salud pública y el acceso y acceso a estos servicios es un derecho legal, sin embargo, se le niega. Conclusión: es posible evidenciar obstáculos para acceder a los servicios del Sistema Único de Salud, principalmente debido a la falta de documentos de identificación, además de la existencia de discriminación al asistir a estos servicios. También hay debilidades en las políticas públicas con respecto a las necesidades de este público, creando injusticias cuando no respetan sus derechos y necesidades. DESCRIPTORES: Población sin hogar; Salud; Salud pública.

RECEBIDO EM: 29/07/2020 APROVADO EM: 31/07/2020

\section{Júlia Gomes Sousa}

Enfermeira. Graduada em Enfermagem pelo Centro Universitário de Juazeiro do Norte- UNIJUAZEIRO (2020).

ORCID: 0000-0001-8054-1066 


\section{Magda Oliveira da Silva}

Enfermeira. Graduada em Enfermagem pelo Centro Universitário de Juazeiro do Norte- UNIJUAZEIRO (2020).

ORCID: 0000-0002-4846-0925

\section{Luciana Nascimento Mascarenhas}

Enfermeira. Graduada em Enfermagem pela pelo Centro Universitário de Juazeiro do Norte- UNIJUAZEIRO (2020).

ORCID: 0000-0001-6228-4764

\section{Hellen Cavalcanti Barbosa}

Enfermeira. Graduada em Enfermagem pelo Centro Universitário de Juazeiro do Norte- UNIJUAZEIRO (2020). Enfermeira Assistencialista na Estratégia Saúde da Família (ESF), Remanso, Orocó-PE. Tem experiência na área de Enfermagem.

ORCID: 0000-0003-0579-3903

\section{Maria de Lourdes de Macedo Bernardo}

Enfermeira. Graduada em Enfermagem pelo Centro Universitário de Juazeiro do Norte- UNIJUAZEIRO (2020).

ORCID: 0000-0002-3505-5453

\section{Dayse Christina Rodrigues Pereira Luz}

Enfermeira, Pós Doutoranda em Ciências da Saúde pelo Centro Universitário de Saúde do ABC - FMABC; Membro do Grupo de Pesquisa em Ciências da Saúde do CNPq Docente do Centro Universitário de Juazeiro do Norte- UNIJUAZEIRO.

ORCID: 0000-0002-5719-3574

\section{INTRODUÇÃO}

A tualmente o colapso enfrentado pela economia e saúde, tem desencadeado um grande número de desemprego, culminando em aumento da listagem de pessoas em situação de rua dispostas a ocupar ambientes urbanos que não são adequados para a moradia, contudo, essa conjuntura engloba uma condição de vida, trabalho e inclusão social de ampla vulnerabilidade ${ }^{1}$.

De acordo com o Decreto Presidencial $n^{\circ} 7.053$ de 23 de dezembro de 2009, a população em situação de rua (PSR) é definida como uma comunidade heterogênea que partilha a extrema pobreza, as conexões familiares quebradas ou enfraquecidas, a ausência de residência convencional regular, e que utiliza os sítios públicos para moradia e meio de sustento, de forma circunstancial ou permanente ${ }^{2}$.

A circunstância de residir na rua estabelece uma condição de grande fragilidade social dessa população, marcada pela inconsistência da qualidade de vida e saúde, exposição a fatores desencadeadores de doenças, violências, ausência de fonte de renda, discriminação relacionada ao acesso a domínios e serviços de saúde e falha da assistência dos direitos humanos e sociais ${ }^{3}$.

Por volta dos anos 2000, o Governo Federal passou a incluir o público residente

\section{A circunstância}

de residir na rua

estabelece uma

condição de grande

fragilidade social

dessa população,

marcada pela

inconsistência da

qualidade de vida e

saúde das ruas em seus regulamentos. As primeiras normativas foram a Política Nacional de Assistência Social, a Lei 11.258, de 30 de dezembro de e o Movimento Nacional da População de Rua em 2005, que expandiu a visibilidade desta população pelo Governo Federal' ${ }^{4}$.

No dia 23 de dezembro de 2009, foi propagada a Política Nacional para a População em Situação de Rua (PNPSR), o principal marco do reconhecimento deste público pelo Estado. Em virtude disso, surgiram os Centros Pop e as equipes de Consultório na Rua, propostos estritamente à este público, com o intuito de concretizar a inclusão social e a garantia de direitos ${ }^{5-6}$.

Diante do exposto, questiona-se: Há dificuldade de acesso aos serviços de saúde pela população em situação de rua? Existem discriminações durante $\mathrm{o}$ atendimento nesses serviços? Logo, destaca-se a relevância do estudo por abordar uma temática que evidencia uma população que tem seus direitos mais básicos negados. Contudo, o objetivo do estudo é analisar o acesso aos serviços de saúde e se há discriminações no atendimento à população em situação de rua nesses serviços.

\section{MÉTODO}

O estudo em questão, trata-se de uma 
revisão sistemática da literatura com metassíntese. Para alcançar a produção científica, foram utilizadas como fonte de busca a Biblioteca Virtual em Saúde (BVS) e as bases de dados: Scientific Electronic Library Online (SciELO), Medical Literature Analysis and Retrieval System Online (MEDLINE), Literatura Latino-americana e do Caribe em Ciências da Saúde (LILACS) e Base de Dados em Enfermagem (BDENF). O levantamento das obras científicas ocorreu entre outubro de 2019 e abril de 2020, utilizados os descritores (DeCS): "população em situação de rua", saúde e "saúde pública", associados ao operador booleano AND, da seguinte forma: "população em situação de rua" AND saúde AND "saúde pública".

Foram incluídos na pesquisa, os estudos que atendiam aos seguintes critérios empregados: $1^{\circ}$ ) artigos disponíveis integralmente, $2^{\circ}$ ) publicados entre os anos de 2015 a 2020, $3^{\circ}$ ) estarem escritos em português, inglês ou espanhol. Foi realizada uma leitura dos títulos e resumos dos materiais encontrados, para selecionar os artigos que se enquadravam nos critérios estabelecidos e foram excluídos os documentos que não possuíam

\section{Figura 1 - Fluxograma de eleição dos estudos.}

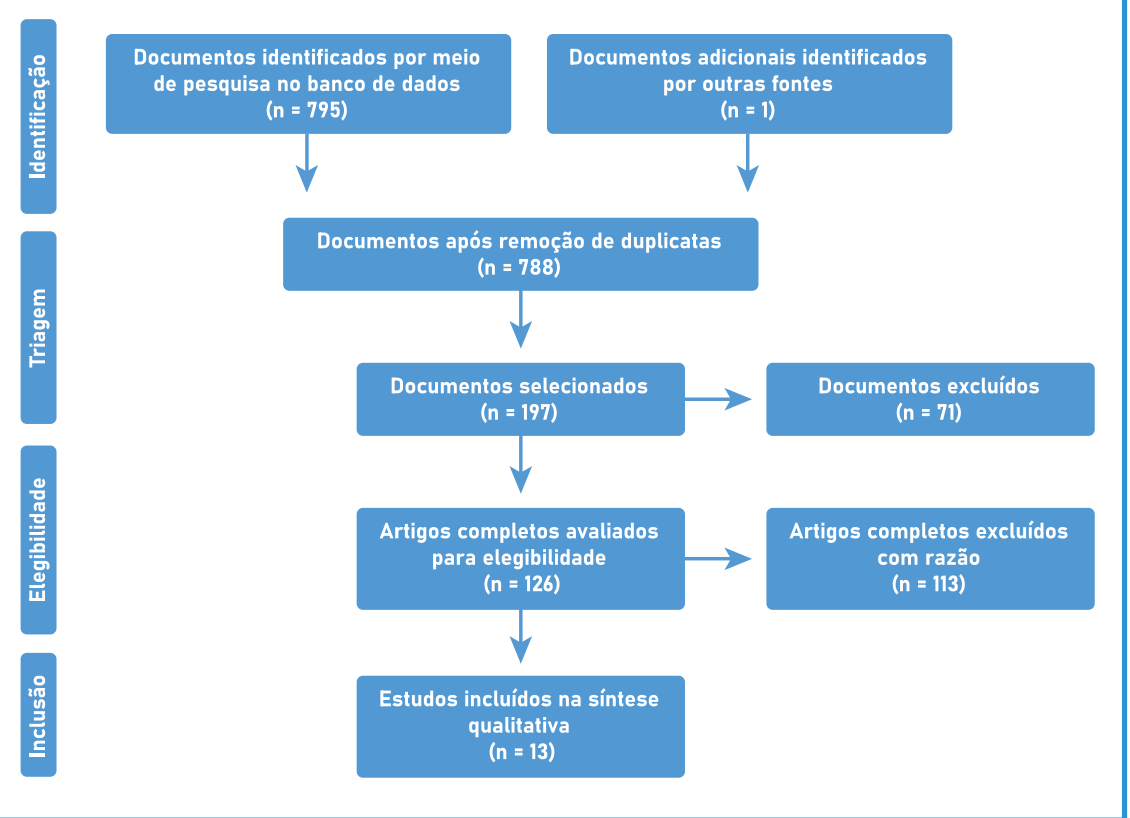

Fonte: Protocolo PRISMA. como ideia central a atenção à população em situação de rua no Sistema Único de Saúde, estudos repetitivos em diferentes bases de dados, dissertações, documentários, monografias, projetos, resumos, relatos e teses.

No que tange aos aspectos éticos da pesquisa para as revisões, não se faz necessário a submissão ao Comitê de ética e pesquisa com seres humanos. Uma vez que, os artigos utilizados já passaram pela a apreciação ética e encontram-se disponíveis online nas bases de dados. Logo, é possível afirmar que todos os preceitos da resolução do Conselho Nacional de Saúde $n^{\circ} 466 / 12$ foram respeitados. se faz necessário

a submissão ao

Comitê de ética e

pesquisa com seres

humanos.

\section{RESULTADOS E DISCUSSÃO}

Ao realizar uma busca com a seguinte combinação de descritores e operador booleano: "população em situação de rua" AND saúde AND "saúde pública" na BVS, foram filtradas apenas as bases de dados MEDLINE, LILACS E BDENF, foi obtido uma totalidade de 744 documentos. Ao realizar a mesma busca na ScieELO, foram encontrados 51 estudos, somando um conjunto de 795 documentos distribuídos na MEDLINE (672); LILACS (62); SciELO (51) e BDENF (10). Após aplicabilidade dos critérios de inclusão e exclusão e somente 13 artigos fizeram parte da construção do estudo.

Com o intuito de demonstrar o processo de busca e escolha dos estudos, foi utilizado um fluxograma baseado no protocolo PRISMA, que consiste em quatro passos (Identifcação, triagem, elegibilidade e inclusão), com intuito de auxiliar os autores em revisões sistemáticas e meta-análises, reunindo estudos semelhantes o qual são avaliados detalhadamente em sua metodologia e são reunidos em uma análise estatística ${ }^{7}$ O fluxograma encontra-se ilustrado na figura 1 .

Ademais, os documentos selecionados estão organizados de acordo com o achado mais recente, para o menos recente. Encontram-se ilustrados na tabela 1 , quanto ao autor e ano de publicação, objetivo e principais achados.

Ao explorar as principais questões encontradas nos artigos contidos nesta revisão, foi possível evidenciar que a população 
Tabela 1 - Distribuição dos artigos quanto ao autor e ano de publicação, objetivo e principais resultados.

\section{Autor e ano}

Objetivo

Tsai J, Michal W Tsai J,

Michal W, 2020

Lira CDG, Justino JMR, Paiva IKS, Miranda MGO, Saraiva AKM, 2019

Wijk LBV, Mângia EF, 2019

Koopmans FF, Daher DV, Acioli S, Sabóia VM, Ribeiro CRB, Silva CSSLD, 2019

Pinto AH, Fermo VC, Peiter CC, 2018

Cardoso AC, Santos DS, Mishima SM, Anjos DSC, Jorge JS, Santana HP, 2018

Sicari AA, Zanella AV, 2018

Oliveira RG, 2018

Oliveira DM, Expedito AC, Aleixo MT, Carneiro NS, Jesus MCP, Merighi MAB, 2018
Avaliar a condição dos sem-teto frente à quarentena.

Ponderar o acesso da população em situação de rua aos serviços de saúde.

Conhecer as ações de saúde dirigidas a PSR; identificar se se respondem às suas necessidades.

Identificar elementos importantes na assistência às pessoas em situação de rua na Atenção Primária à Saúde

Apresentar as experiências de uma enfermeira na sua prática em uma equipe de Consultórios na Rua.

Analisar fundamentos do método de trabalho nos consultórios na rua, destacando os desafios e as da assistência aos moradores de rua.

Investigar a característica das pessoas que vivem em situação de rua.

Identificar as práticas de saúde em contextos de vulnerabilização e a atenção à saúde de pessoas em situação de rua.

Compreender as necessidades, expectativas e produção de cuidados das pessoas em situação de rua.

\section{Principais Resultados}

Pessoas em situação de rua são um grupo vulnerável e seu potencial de exposição ao COVID-19 pode afetar negativamente sua saúde mental e física. Podendo aumentar as taxas de contágio e mortalidade pela doença por não haver espaço adequado para obedecer a quarentena e pela dificuldade de acesso aos serviços de saúde.

O acesso aos serviços de saúde pela PSR ocorre comumente, através dos serviços de urgência e emergência de forma rápida e sem vínculo. Em relação a dificuldades no acesso, existe a burocratização, decorrente da falta do cartão SUS e de endereço fixo.

As equipes de Consultório na Rua e os CAPS realizam o que é preconizado de forma parcial, pois existem dificuldades em realizar o trabalho em rede respondendo as urgências, sugerindo deficiência na integração dos pontos da rede de cuidado e falta de corresponsabilização.

A construção do cuidado à PSR pode acontecer na Atenção Primária a Saúde. E os elementos-chave para a produção desse cuidado são o vínculo, a escuta qualificada, a compreensão do sujeito, a identificação de necessidades, tornando o cuidado de forma longitudinal.

Foram visualizadas ocorrências de rejeição da população em situação de rua pela sociedade, inclusive por profissionais de saúde, barreiras para acessar à Rede de Atenção à Saúde por essas pessoas de rua, injustiças sociais e brechas nas políticas públicas e sua inserção na prática.

Há uma forte vulnerabilidade em que os beneficiários dos serviços estão inseridos, no que tange as necessidades básicas, A maioria não tem garantia de higiene, alimentação moradia, segurança pública e também de participar do programa de assistência social, ou seja, possuem direitos negados. Outra necessidade de saúde identificada foi o ingresso aos setores da Rede de Atenção à Saúde, que muitas vezes é dificultado pela falta de serviços e principalmente, por deficiência no acolhimento e recepção.

A população em situação de rua é integrada por homens em sua maioria, foi evidenciado o uso patológico de álcool, falta de renda, a maioria apresentou pelo menos uma doença crônica, e alguns afirmaram que se percebem velhos antes dos 60 anos, decorrente do meio social e à qualidade precária de vida.

A universalidade do SUS, tem implicado uma demarcação socioeconômica e territorial de políticas de saúde, em particular da atenção primária. A Estratégia Saúde da Família em teoria, engloba toda a população brasileira. Contudo, como as políticas de saúde são sistematizadas por prioridades, a Estratégia de Saúde da Família tem sido voltada para os territórios onde vivem as pessoas menos favorecidas, pois geralmente apresentam, os piores indicadores de saúde.

As carências das pessoas em situação de rua estão associadas à segurança, alimentação, sono, descanso e acesso aos serviços de saúde. A produção de cuidados ocorre através de redes de apoio criadas para atender às necessidades biológicas, espirituais e sociais-afetivas. As expectativas desse grupo social são retratadas pelo desejo de que os serviços de saúde atendam às suas necessidades e considerem suas particularidades. 


\section{artigo}

Sousa, J.G.; Oliveira da Silva, M.; Mascarenhas, L.N.; Barbosa, H.C.; Bernardo, M.L.M.; Luz, D.C.R.P.

Atenção à população em situação de rua no âmbito do sistema único de saúde: uma revisão sistemática com metassíntese

Paiva IKS, Lira CDG, Justino

JMR, Miranda MGO, Saraiva

AKM, 2016

Barata RB, Carneiro JN, Ri-

beiro MCA, Silveira C, 2015

Luz LCX, 2015
Conhecer a produção científica brasileira sobre pessoas que vivenciam situações de rua sob o olhar da saúde.

Levantar dados sobre a caracterização da PSR, suas necessidades e as políticas criadas para atendê-la.

Delinear o perfil e o acesso a serviços, da população em situação de rua.

Analisar a questão social no Brasil, com ênfase na população em situação de rua do Rio de Janeiro.
Foram identificadas dificuldades no acesso aos serviços de saúde pelos moradores de rua, no que tange à sua inclusão no sistema de saúde brasileiro, a hostilidade relativa às condições de higiene, falta de documentação, discriminação pela inaptidão física e mental e preconceito racial. Contudo, a saúde é comprometida pela violação dos princípios da universalidade do acesso aos serviços de saúde, equidade e atenção integral.

As pessoas que habitam as ruas são heterogêneas e possuem condições de vida diferentes. Essas características demandam cuidados específicos, relacionados a saúde física, mental e necessidades humanas essenciais. Nas políticas de saúde, persistem os problemas de acesso, desarticulação de setores e ainda a assistência medicalizante.

Foi evidenciado que grande parcela do grupo em situação de rua considerou sua saúde regular, ruim ou muito ruim, considerando suas precárias condições de vida. Dentre os que procuram os serviços de saúde, 95\% são atendidos, demonstrando que, apesar das barreiras, os mesmos obtiveram acesso.

As peculiaridades da situação da população em situação de rua, implicam um posicionamento abrangente. Portanto, responder às demandas desse grupo social exige expandir ações relacionadas à garantia de seus direitos, como condição humana, trabalho, assistência social, desenvolvimento urbano, educação, proteção, nutrição, saúde e cultura.

Fonte: Elaborado pela autora.

em situação de rua sofre rejeição no atendimento aos serviços públicos de saúde e o acesso de entrada e constância nesses serviços, é um direito legal, contudo, está sendo negado. Eles têm garantia de entrada, mas a continuidade da assistência, está sendo omitida. Os serviços mais utilizados pelos mesmos são a urgência e emergência por apresentarem uma resposta imediata às suas queixas em saúde. Além disso, a falta de documentos e cartão do SUS, a falta de residência convencional e a tendência migratória, dificulta a definição de território e o acesso à atenção básica de saúde, quebrando assim a continuidade do cuidado ${ }^{5-9-11-14}$.

Similarmente os grupos de Consultório na Rua e os Centros Atenção Psicossocial, compreendem serviços voltados ao cuidado da população em situação de rua, sobretudo os Consultórios na Rua ${ }^{10}$. Embora haja investimentos, existem limites nesse processo em relação à burocratização dos serviços diante das necessidade dessas pessoas ${ }^{8}$. É de suma importância instituir vínculo com os pacientes e conexões intersetoriais, aumentando a articulação em vários níveis assistenciais, produzindo cuidados efetivos, valorizando o atendimento humanizado ${ }^{2-13}$.

Discernir as necessidades de saúde da PSR, demanda a mudança do paradigma centrado na doença, como único meio de lidar com as queixas expostas por eles. Essas carências não se restringem somente ao aspecto biológico, mas envolvem as vulnerabilidades dos indivíduos. Considerando que essa população é marcada por condições de vida difíceis, falta de acesso aos direitos básicos como saúde, educação e segurança, a qualidade de vida é implementada ao oferecer meios para dar mais dignidade à vida ${ }^{1-3-12}$.

Embora enfrentem dificuldades, a população em situação de rua obtém acesso aos serviços de saúde, ainda que seja apenas para alívio da condição atual ${ }^{4}$. Trabalhar com essa população, requer a expansão de ações que não se limitem apenas a saúde-doença, mas também à garantia de seus direitos de trabalho, assistência social, e desenvolvimento. É como atuam os Centros POP, que são espaços de amparo e proteção, realizam a reinserção social desse público, além de ofertar alimentação, espaço para higiene pessoal e rodas de conversa acerca da saúde, educação e cultura ${ }^{46}$.

\section{CONCLUSÃO}

Diante dos dados apresentados, é possível identificar que a população em situa- 
ção de rua enfrenta obstáculos para acessar os serviços no âmbito do Sistema Único de Saúde, principalmente na atenção básica, sobretudo por falta de documentos de identificação, o que permite o rompimento da integralidade da assistência à saúde, além de sofrer discriminações durante o atendimento nesses serviços.

Há ainda fragilidades nas políticas públicas referentes às necessidades deste público, que geram injustiças ao desrespeitar seus direitos. É de suma importância a ressignificação da execução das políticas, legislações e protocolos de acolhimento à essa população.
Contudo os Consultórios na Rua são uma ferramenta com potencial para transformar a realidade dessa população no Brasil, fortalecendo a cidadania desses grupos, oferecendo um atendimento humanizado, com respeito à dignidade e considerando a singularidade de cada ser.

\section{REFERÊNCIAS}

1. Tsai J, Michal W. COVID-19: potential public health problem for homeless populations. [Internet]. 2020. Disponível em: < https://www.thelancet.com/journals/lanpub/ article/PIIS2468-2667(20)30053-0/fulltext>. https://doi. org/10.1016/ S240268-2667(20)30053-0.

2. Wijk LBV, Mângia EF. Atenção psicossocial e o cuidado em saúde à população em situação de rua. Ciênc. saúde coletiva [Internet]. 2019; 24(9): 3357-3368. Disponivel em: <http://www.scielo.br/scielo.php?script=sci_arttext\&pid=S1413-81232019000903357\&lng=pt>. Epub 09-Set-2019. https://doi.org/10.1590/141381232018249.29872017.

3. Sicari AA, Zanella AV. Pessoas em Situação de Rua no Brasil: Revisão Sistemática. Psicologia: Ciência e Profissão. Brasília, v. 38, n. 4, p. 662-679. 2018. Disponível em: <http://www.scielo.br/scielo.php?script=sci_arttext\&pi$\mathrm{d}=\mathrm{S} 1414-98932018000500662$ \&lng=en\&nrm=iso >. https://doi.org/10.1590/1982-3703003292017.

4. Barata RB, Carneiro JN, Ribeiro MCA, Silveira C. Desigualdade social em saúde na população em situação de rua na cidade de São Paulo. Saude soc. [Internet]. 2015; 24 (Suppl 1): 219232. Disponivel em: <http://www.scielo.br/scielo.php?scrip$\mathrm{t}=\mathrm{sci}$ _arttext\&pid=S0104-12902015000500219\&lng=pt>. https://doi.org/10.1590/s0104-12902015s01019.

5. Paiva IKS, Lira CDG, Justino JMR, Miranda MGO, Saraiva $A K M$. Direito à saúde da população em situação de rua: reflexões sobre a problemática. Ciênc. saúde coletiva [Internet]. 2016; 21(8): 2595-2606. Disponivel em: <http://www.scielo.br/scielo.php?script=sci_arttext\&pid=S1413-81232016000802595\&Ing=pt>. https://doi. org/10.1590/1413-81232015218.06892015.

6. Luz LCX. Políticas para a população adulta em situação de rua: questões para debate. Florianópolis, v. 18, n. 1, p. 7485, jan/jun. 2015. Disponível em: <http://www.scielo.br/pdf/ rk/v18n1/1414-4980-rk-18-01-00074.pdf>.

7. Galvão TF, Pansani TSA, Harrad D. Principais itens para relatar Revisões sistemáticas e Meta-análises: A recomendação PRISMA. Epidemiol. Serv. Saúde [Internet]. 2015; 24(2): 335342. Disponível em: <http://www.scielo.br/scielo.php?scrip$\mathrm{t}=\mathrm{sci}$ _arttext\&pid=S2237-96222015000200335\&lng=en>. https://doi.org/10.5123/S1679-49742015000200017.

8. Lira CDG, Justino JMR, Paiva IKS, Miranda MGO, Saraiva $A K M$. $O$ acesso da população em situação de rua é um direito negado? REME - Rev Min Enferm. [Internet].
2019; 23:e-1157. Disponivel em: <https://pesquisa.bvsalud.org/portal/resource/pt/biblio-1004990>. https://doi. org/10.5935/1415-2762.20190004.

9. Koopmans FF, Daher DV, Acioli S, Sabóia VM, Ribeiro CRB, Silva CSSLD. Living on the streets: An integrative review about the care for homeless people. Rev Bras Enferm. [Internet]. 2019;72(1):211220. Disponivel em < https://www.scielo.br/scielo.php?script=sci_arttext\&pi$\mathrm{d}=$ S0034-71672019000100211\&tIng=en>. https://doi. org/10.1590/0034-7167-2017-0653.

10. Pinto AH, Fermo VC, Peiter CC, Fernandes VMB, Heideman ITSB. Determinantes sociais, equidade e consultório na rua. Rev. enferm. UFPE on line; [Internet]. 12(12): 35133520, dez. 2018. Disponível em: <https://pesquisa.bvsalud. org/portal/resource/pt/biblio-1005383>. https://doi.org/10.5205/1981-8963-v12i12a236025p3513-3520-2018.

11. Cardoso AC, Santos DS, Mishima SM, Anjos DSC, Jorge JS, Santana HP. Challenges and potentialities of nursing work in street medical offices. Rev. Latino-Am. Enfermagem [Internet]. 2018; 26: e3045. Disponível em: <http:// www.revenf.bvs.br/scielo.php?script=sci_arttext\&pi$\mathrm{d}=$ S0104-11692018000100358\&Ing=pt>. Epub 11-Out 2018. http://dx.doi.org/10.1590/1518-8345.2323.3045.

12. Oliveira RG. Práticas de saúde em contextos de vulnerabilização e negligência de doenças, sujeitos e territórios: potencialidades e contradições na atenção à saúde de pessoas em situação de rua. Saúde soc. [Internet]. 2018; 27(1): 3750. Disponivel em: <http://www.scielo.br/scielo.php?script=sci_arttext\&pid=S0104-12902018000100037\&Ing=en>. http://dx.doi.org/10.1590/s0104-12902018170915.

13. Oliveira DM, Expedito AC, Aleixo MT, Carneiro NS, Jesus MCP, Merighi MAB. Necessidades, expectativas e produção de cuidados de pessoas em situação de rua. Rev. Bras. Enferm. [Internet]. 2018; 71 (Suppl 6): 2689-2697. Disponível em: <http://www.scielo.br/scielo.php?script=sci_arttext\&pid=S0034-71672018001202689\&Ing=en>. https://doi. org/10.1590/0034-7167-2017-0612.

14. Hino P, Santos JO, Rosa AS. Pessoas que vivem na rua do ponto de vista da saúde. Rev. Bras. Enferm. [Internet]. 2018; 71 (Supl. 1): 684-692. Disponível em: <http://www.scielo.br/scielo.php?script=sci_arttext\&pid=S0034-71672018000700684\&lng=en>. http://dx.doi. org/10.1590/0034-7167-2017-0547. 